Provided for non-commercial research and education use. Not for reproduction, distribution or commercial use.

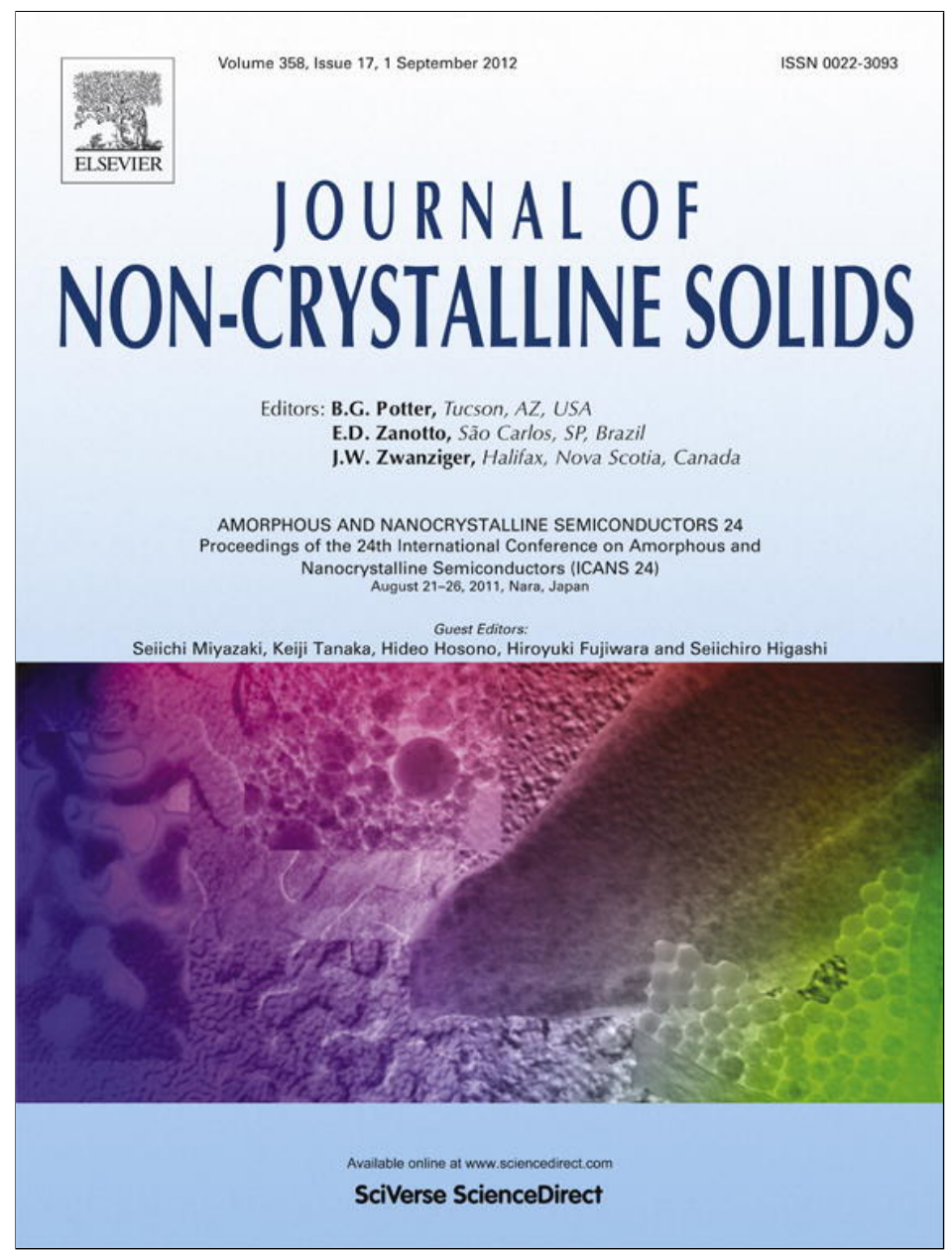

This article appeared in a journal published by Elsevier. The attached copy is furnished to the author for internal non-commercial research and education use, including for instruction at the authors institution and sharing with colleagues.

Other uses, including reproduction and distribution, or selling or licensing copies, or posting to personal, institutional or third party websites are prohibited.

In most cases authors are permitted to post their version of the article (e.g. in Word or Tex form) to their personal website or institutional repository. Authors requiring further information regarding Elsevier's archiving and manuscript policies are encouraged to visit:

http://www.elsevier.com/copyright 


\title{
Charge collection in amorphous silicon solar cells: Cell analysis and simulation of high-efficiency pin devices
}

\author{
Michael Stuckelberger*, Yannick Riesen, Brice Perruche, Matthieu Despeisse, \\ Nicolas Wyrsch, Christophe Ballif \\ Ecole Polytechnique Fédérale de Lausanne (EPFL), Institute of Microengineering (IMT), Photovoltaics and Thin Film Electronics Laboratory, Rue Breguet 2, CH-2000 Neuchâtel, Switzerland
}

\section{A R T I C L E I N F O}

\section{Article history:}

Received 21 August 2011

Received in revised form 1 November 2011

Available online 14 December 2011

\section{Keywords:}

Thin film silicon solar cells;

a-Si:H:

Electric field deformation;

Charge collection;

Charged dangling bonds

\begin{abstract}
A B S T R A C T
The drift length $\mathrm{L}_{\text {drift }}=\mu \tau E$ within the $i$ layer of a-Si:H solar cells is a crucial parameter for charge collection and efficiency. It is strongly reduced not only by light-induced reduction of $\mu \tau$, but also by electric field deformation $\Delta E$ by charges near the $p-i$ and $i-n$ interfaces. Here, a simple model is presented to estimate contributions of free carriers, charges trapped in band tails and charged dangling bonds to $\Delta E$. It is shown that the model reproduces correctly trends observed experimentally and by ASA simulations: charged dangling bonds contribute most to $\Delta E$ of meta-stable cells. Electrons trapped in the conduction band tail near the $i-n$ interface lead to the strongest field deformation in the initial state, while positively charged dangling bonds near the $p-i$ interface get more important with degradation under AM1.5g spectrum. The measurable parameter $V_{\text {coll }}$ is proposed as an indirect parameter to estimate the electric field, and an experimental technique is presented that could enable the distinction of defects near the $p-i$ and the $i-n$ interfaces.
\end{abstract}

(c) 2011 Elsevier B.V. All rights reserved.

\section{Introduction}

One of the main efficiency limitations in hydrogenated amorphous silicon (a-Si:H) based solar cells remains to be the electrical performance degradation arising from metastable defect creation in the form of dangling bonds under light exposure (Staebler-Wronski-Effect (SWE) [1]). These defects reduce the mobility lifetime product $\mu \tau$ and, with it, the drift length $L_{\mathrm{drift}}=\mu \tau E$. As the cells are drift driven, this affects seriously the charge collection and efficiency of the cells.

With a degraded mobility-lifetime product, the strength of the electric field within the absorber layers gets crucial for the preservation of a sufficiently high charge extraction from the intrinsic ( $i$ ) layer. In addition, charges trapped not only in the doped $p$ and $n$ layers, but also in $i$ layers lead to a deformation of the electric field $\Delta E$. Using for simulations and deposited cells in the following always the same doped layers, we concentrate on the $i$ layer and discuss in this article the influence of (a) free carriers, (b) charges trapped in valence and conduction band tails, and (c) charged dangling bonds on the electric field and hence on the charge collection. As the electric field within solar cells is experimentally hardly accessible, results from a very simple instructive model are compared with simulations with the ASA program [2] and experiments, including the measurement of parameters that allow to deduce information about the electric field within the $i$ layer.

\footnotetext{
* Corresponding author.

E-mail address: michael.stuckelberger@epfl.ch (M. Stuckelberger). URL: http://pvlab.epfl.ch (M. Stuckelberger).
}

One of these parameters is the measurable collection voltage $V_{\text {coll }}$ as discussed in [3-5]. It obtains its physical meaning by the link to the collection function or -efficiency $\chi$ that is defined as:

$\chi=\frac{Q_{\text {coll }}}{Q_{\text {photo }}}=\frac{J_{\text {coll }}}{J_{\text {photo }}}=1-\frac{\int R(x) d x}{\int G(x) d x}$,

with $Q_{\text {coll, photo }}, J_{\text {coll, photo }}$ the collected and photogenerated charge and current densities; $R$ and $G$ are the $e^{-} / h^{+}$-pair recombination and generation rates. Different terms for $\chi$ have been derived in literature for various recombination models [3,6-9]. In all these cases, the fractional collection losses (in percent of the generated current) do not depend on $G$ and are therefore proportional to $J_{\text {photo }}$. Describing the solar cell by the simple equivalent circuit [3], between high illumination regime with dominant series resistance losses and low illumination regime with dominant parallel resistance losses, there exists a medium illumination regime, where the recombination term is dominant. In this regime, one finds independently of the form of $\chi$ the collection voltage

$\left.\left.J_{\mathrm{sc}} \cdot R_{\mathrm{sc}} \approx \chi\right|_{V=0} \cdot \frac{\partial V}{\partial \chi}\right|_{V=0} \doteq V_{\text {coll }}$

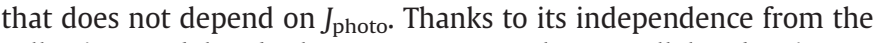
collection model and other parameters such as parallel and series resistance, $V_{\text {coll }}$ is a good parameter for intrinsic charge collection that can provide additional information to e.g. quantum efficiency or fill 
factor [10]. In a more intuitive approach, $V_{\text {coll }}$ is the voltage that must be applied to the equivalent ohmic resistance $R_{\mathrm{sc}}$ for a current $J_{\mathrm{sc}}$ to flow.

\section{Simple model to estimate $\Delta E(x)$}

The main assumptions for this model are: (1) No recombination of holes and electrons in the $i$ layer and (2) diffusion is negligible as charge transport mechanism compared to drift. Starting from a constant nominal electric field $E_{\text {nom }}=\frac{V_{\text {bi }}}{d_{i}}$ with the built-in voltage $V_{\mathrm{bi}}$ and the $i$ layer thickness $d_{i}$, charge profiles and the electric field deformation $\Delta E=E-$ $E_{\text {nom }}$ caused by such charges are calculated without iteration of the transport equations (limiting this model to cases, in which $\Delta E \ll E$ ). The aim of such crude simplifications is to provide intuitive insights in the physical processes of charge collection and explain measured trends.

The model is based on the intuitively plausible model of amphoteric defect states that can be charged positively $\left(D^{+}\right)$, negatively $\left(D^{-}\right)$or remain neutral $\left(D^{0}\right)$. As derived in [11], these states are occupied with the probabilities $f^{+}, f^{-}$, and $f^{0}$ which depend on the capture cross sections $\sigma_{\mathrm{p}, \mathrm{n}}^{+, 0,-}$ of $D^{+}, D^{-}$and $D^{0}$ for holes and electrons and on the free carrier concentrations $p_{\mathrm{f}}$ and $n_{\mathrm{f}}$. With assumption (1), we get the particle fluxes $\Phi_{\mathrm{p}, \mathrm{n}}(x)$ at position $x$ in an $i$ layer between $x=0(p-i$ interface) and $x=d_{i}$ :

$\Phi_{\mathrm{p}}(x)=\int_{x}^{d_{i}} G(\xi) d \xi \quad$ and $\quad \Phi_{\mathrm{n}}(x)=\int_{0}^{\mathrm{x}} G(\xi) d \xi$.

With assumption (2), it is:

$p_{\mathrm{f}}, n_{\mathrm{f}}(x)=\frac{\Phi_{\mathrm{p}, \mathrm{n}}(x)}{v_{\mathrm{p}, \mathrm{n}}^{\mathrm{th}}}=\frac{\Phi_{\mathrm{p}, \mathrm{n}}(x)}{\mu_{\mathrm{p}, \mathrm{n}} \cdot E_{\mathrm{nom}}}$

with the thermal velocities $v^{\text {th }}$. Based on [12,13], we assume further $\sigma^{ \pm} \equiv \sigma_{\mathrm{p}}^{-}=\sigma_{\mathrm{n}}^{+}$and $\sigma^{0} \equiv \sigma_{\mathrm{p}}^{0}=\sigma_{\mathrm{n}}^{0}$ and write $\zeta \dot{\equiv} \frac{\sigma^{ \pm}}{\sigma^{0}}, \quad \gamma \dot{\frac{\mu_{\mathrm{n}}}{\mu_{\mathrm{p}}}}=\frac{v_{\mathrm{n}}^{\mathrm{th}}}{v_{\mathrm{p}}^{\mathrm{th}}}$, and $\eta(x) \doteq \frac{\Phi_{\mathrm{n}}(x)}{\Phi_{\mathrm{p}}(x)}$. For numerical calculations, we took $\zeta=50$ and $\gamma=3$ $[13,14]$. The occupation functions reduce then to:

$f^{+}(x)=\frac{1}{1+\zeta \eta(x)+\eta^{2}(x)}, \quad f^{-}(x)=\frac{\eta^{2}}{1+\zeta \eta(x)+\eta^{2}(x)}$.

Considering always only one type of charge carriers, the field deformation relative to the $p-i$ (index $p$, considering holes) or to the $i-n$ interface (index $n$, considering electrons), is calculated as:

$\Delta E_{p, n}(x)=\frac{q}{\epsilon_{0} \epsilon_{\mathrm{r}} \int_{0, x}^{x, d_{\mathrm{i}}} N_{p, n}\left(x^{\prime}\right) d x^{\prime}}$

Depending on the charge type, $N_{p, n}\left(x^{\prime}\right)$ is either the free charge carrier concentration $p_{\mathrm{f}}, n_{\mathrm{f}}$ calculated with Eq. (4), or the concentration of charge carriers trapped in band tails $p_{\mathrm{bt}}, n_{\mathrm{bt}}=\frac{p_{\mathrm{f}}}{\Theta_{p}}, \frac{n_{\mathrm{f}}}{\Theta_{n}}$ (with the Rose trapping factors $\Theta_{p} \approx 0.005$ and $\Theta_{n} \approx 0.1$ ), or the concentration of charged dangling bonds $p_{\mathrm{db}}, n_{\mathrm{db}}=f^{+,-} \cdot N_{\mathrm{db}}$ with the dangling bond concentration $N_{\mathrm{db}}$. The choice of the remaining parameters $G(x)$ and $N_{\mathrm{db}}(x)$ is principally the same as in the ASA simulations (see Section 3 ) and will be discussed in Sections 4 and 5.

From $\Theta_{p, n} \ll 1$ follows directly that $\Delta E_{p, n}$ caused by free carriers can be neglected with respect to $\Delta E_{p, n}^{\mathrm{bt}}$.

\section{Experimental}

An $i$ layer thickness series $(100-1000 \mathrm{~nm})$ of $\sim 0.25 \mathrm{~cm}^{2}$ a-Si:H cells was deposited by PECVD in superstrate configuration on glass with $2 \mu \mathrm{m}$ thick boron-doped zinc oxide (LPCVD) TCO layers on both sides (a second series deposited in another system lead to very similar results). Typical cell efficiencies are above $10 \%$ in initial state and above $8 \%$ stable.

Beside standard $J(V)$-curve measurements for the fill factor $(F F)$, external quantum efficiency ( $\mathrm{EQE}$ ) measurements were used to determine $V_{\text {coll }}$ : Varying the bias voltage $V, V_{\text {coll }}$ can be determined as $V_{\text {coll }}=\left.E Q E(O V) \cdot \frac{\partial V}{\partial E Q E}\right|_{O V}$ (details can be found in [4]). For the present study, $\operatorname{EQE}(V)$ was determined with a probe beam at $420 \mathrm{~nm}$ and blue bias light that entered with the probe beam either from $p$ or $n$ side into the cell. This measurement is therefore sensitive mainly to charge collection near the $p-i$ and $i-n$ interface. [The absorption lengths of the probe beam at $420 \mathrm{~nm}$ and of the blue bias light are in the order of 25 and $65 \mathrm{~nm}$, respectively.]

The ASA package (version 5 [2]) with the optical model GenPro3 was used for simulations. For the simulation parameters we took values obtained from layer measurements, where available, and reasonably chosen values otherwise.

\section{Results}

The correlation between $V_{\text {coll }}$ and $F F$ is discussed in [10,12]. Here, $V_{\text {coll }}$ determined from EQE measurements is compared to the electric field deformation resulting from the simple model, and to a more precise simulation of the deposited cells (see Section 3 ) by the ASA package.

With the creation of dangling bonds by SWE during light soaking, field deformation by charges within the $i$ layer reduces the charge collection additionally to the effect of new recombination centers: In ASA simulation, the dangling bond concentration in the $i$ layer in initial state was set constant to $N_{\mathrm{db}}^{\mathrm{ini}}(x)=3 \cdot 10^{15} \mathrm{~cm}^{-3}$. We simulate the degradation by a linear increase of $N_{\mathrm{db}}$ with $G(x)$ to $N_{\mathrm{db}}^{\mathrm{deg}}(x)=N_{\mathrm{db}}^{\text {ini }}+c \cdot G(x)$ in the degraded state (we assumed $c=2.5 \cdot 10^{-5} \mathrm{~S}$ ). [In the simple model, $N_{\mathrm{db}}$ is chosen 10 times smaller to reproduce qualitatively trends observed, without getting unphysically high $\Delta E$ that would no more justify the assumption $\Delta E \ll E$.] The ASA simulation of charge densities before and after light soaking is shown in Fig. 1. Fig. 2 shows the effect of these charges (ASA simulation and simple model calculations) on the electric field deformation.

The increased $\Delta E$ in the degraded state is confirmed by a decrease of $V_{\text {coll }}$ after light soaking that can be seen in experiment (Fig. 3a) and ASA simulation (Fig. 3c). Not surprisingly, $V_{\text {coll }}$ is smaller for $n$ side illumination, as the slow holes have to cross the whole $i$ layer and recombine more often than the electrons in the opposite case. To get a feeling for the voltage dependence of the charge collection, the parameter $V^{\prime}$ coll $\left.\dot{\doteq} \frac{\partial V}{\partial \chi}\right|_{V=0}=\left.\frac{\partial V}{\partial E Q E}\right|_{V=0}$ (i.e. $V_{\text {coll }}$ without normalization), has been investigated (see Fig. 3b). Interestingly, it shows a different behavior than $V_{\text {coll. }}$ Namely, it increases strongly for thick cells, when illuminated from $n$ side. The ASA simulation in Fig. $3 d$ proves that this is not just a measurement artifact.
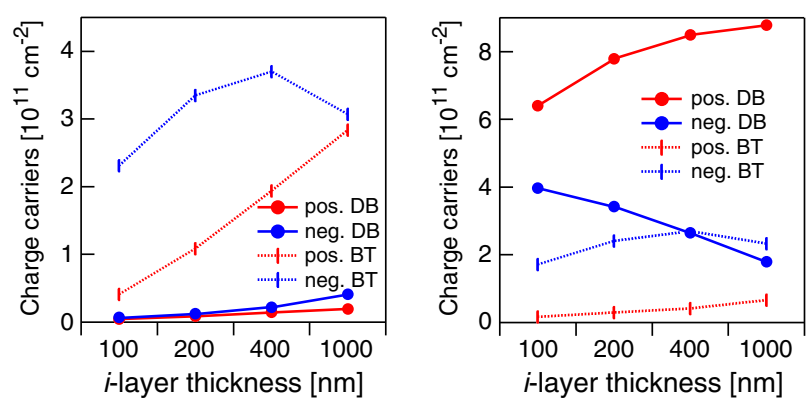

Fig. 1. ASA simulation of charge densities (integrated over whole $i$-layer thickness) in form of trapped charges in band tails (neg. BT for conduction band, pos. BT for valence band) and charged dangling bonds (DB) in initial (left) and degraded state (right) for cells with 100 to $1000 \mathrm{~nm}$ thick $i$-layers. 

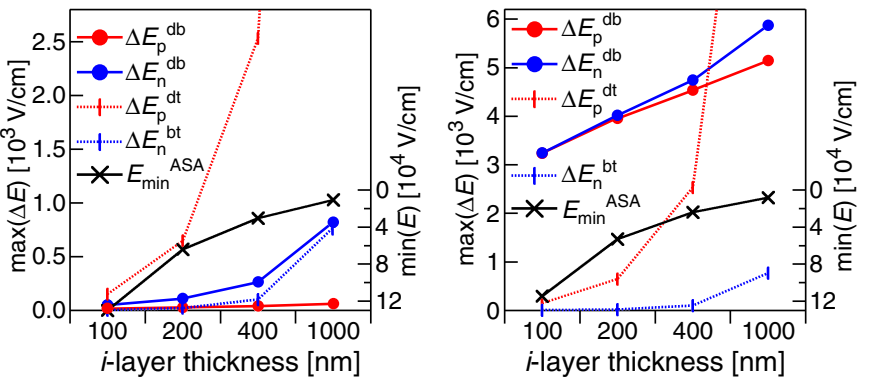

Fig. 2. Estimation of the field deformation $\Delta E$ due to positive $(p)$ and negative $(n)$ charges in band tails (bt) and dangling bonds (db) with the simple model in initial (left) and degraded state (right). $E_{\min }$ is the minimum electric field within the $i$ layer from the corresponding ASA simulations.

\section{Discussion}

While $\Delta E$ is dominated by the $i-n$ interface in initial state (for constant $N_{\mathrm{db}}$ ), the light induced increase of defect states mainly near the $p-i$ interface strongly increases the contribution of the $p-i$ interface to $\Delta E$, as could be seen in Fig. 1: Most $e^{-} / h^{+}$-pairs are created near the $p-i$ interface and the electrons need to travel much further, thus the mean electron flux $\overline{\Phi_{n}}(x)$ is much higher than the mean hole flux $\overline{\Phi_{p}}(x)$, over compensating the lower mobility of holes and leading to a higher electron concentration and dominating therefore $\Delta E$ following Eq. (6).

After light soaking, most defect states near the $p-i(i-n)$ interfaces are, like before, in $D^{+}\left(D^{-}\right)$state and deform the field. There are now more defects to be positively charged near the $p-i$ interface. These lead to a larger electric field deformation than negatively charged dangling bonds near the $i-n$ interface. [In actual solar cells, this effect is even more pronounced, as the $p-i$ interface is already in initial state more defective than the $i-n$ interface.]

Comparing the simple model (Fig. 2) with ASA simulation (Fig. 1), the effect of holes trapped in valence band tail seems to be strongly overestimated as compared to the electrons trapped in conduction band tail; this suggests that the ratio $\frac{\Theta_{p}}{\Theta_{n}}$ as given in [13] and used
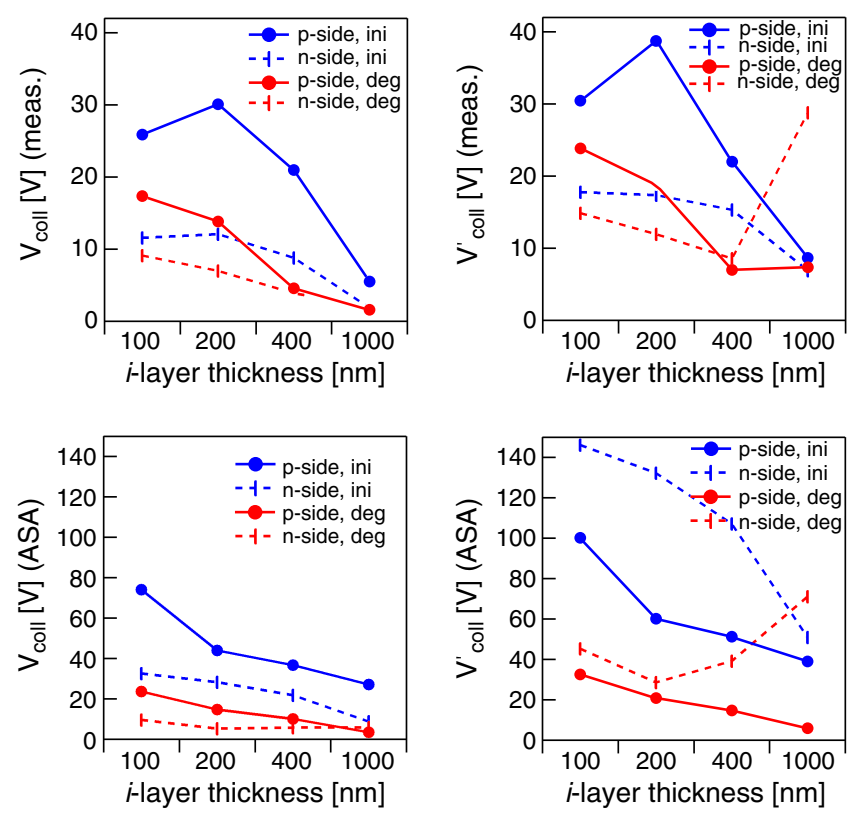

Fig. 3. $V_{\text {coll }}$ and $V_{\text {coll }}^{\prime}=V_{\text {coll }} \cdot E Q E^{-1}(0 V)$ determined from EQE measurements (top) and ASA simulation (bottom) with probe beam at $420 \mathrm{~nm}$ incident with blue bias light from $p$ and $n$ side for cells in initial state and after light soaking. for the simple model calculations might be underestimated. The very strong maximum field deformation for cells with thick absorber layers shows the limitation of non-iteration in the simple model, where the error gets larger, the more $\Delta E$ is approaching the electric field.

A comparison of electric field and charge distributions from ASA simulations (not shown here) shows that the different behavior under $p$ and $n$ side illumination is a field deformation effect that is directly caused by the spatial separation of defects and $e^{-} / h^{+}$-pair generation in case of $n$ side illumination (coincidence for $p$ side illumination). Therefore, $V^{\prime}$ coll could be a parameter that allows getting insight into the distribution of defects in the $i$ layer of working solar cells - this will have to be studied further in detail.

\section{Conclusion}

A simple model has been presented to estimate the electric field deformation in $i$ layers of a-Si:H solar cells, caused by charges trapped in valence and conduction band tails as well as by charged dangling bonds. It has been compared to ASA simulations and measurements on an $i$ layer series of deposited solar cells, where its good ability to describe observed trends, but also its limitations could be seen. It has been shown that field deformation is mainly governed by the $i-n$ interface in the initial state, but that the $p-i$ interface gets more important with light soaking from $p$ side. Further, the use of the collection voltage $V_{\text {coll }}$ and of the derived parameter $V_{\text {coll }}^{\prime}$ has been studied for an evaluation of the charge collection and internal electric field in the $i$ layer.

\section{Acknowledgments}

We acknowledge Swiss Federal Office of Energy (SFOE) for financial support (project no. 101191). Special thanks go to Adrian Billet and Mathieu Charrière for help in cell deposition and measurements.

\section{References}

[1] D.L. Staebler, C.R. Wronski, Reversible conductivity changes in discharge-produced amorphous Si, Appl. Phys. Lett. 31 (1977) 292-294.

[2] B.E. Pieters, J. Krc, M. Zeman, Advanced Numerical Simulation Tool for Solar Cells - ASA5, Conf. Rec. 4th WCPEC, 2, 2006, pp. 1513-1516.

[3] J. Merten, J.M. Asensi, C. Voz, A.V. Shah, R. Platz, J. Andreu, Improved Equivalent Circuit and Analytical Model for Amorphous Silicon Solar Cells and Modules, IEEE Trans. Electron Devices 45 (1998).

[4] C. Hof, Thin Film Solar Cells of Amorphous Silicon: Influence of i-Layer Material on Cell Efficiency, Ph.D. thesis, University Neuchâtel, IMT, 2000.

[5] F. Sculati-Meillaud, Microcrystalline silicon solar cells: Theory, diagnosis and stability, Ph.D. thesis, Université de Neuchâtel, 2006.

[6] K. Hecht, Zum Mechanismus des lichtelektrischen Primärstromes in isolierenden Kristallen, Z. Phys. 66 (1932) 235-245.

[7] R.S. Crandall, Modeling of thin film solar cells: uniform field approximation, J. Appl. Phys. 54 (1983) 7176-7186.

[8] B. Rech, Solarzellen aus amorphem Silizium mit hohem stabilem Wirkungsgrad, Ph.D. thesis, Forschungszentrum Jülich, 1997.

[9] J. Hubin, A. Shah, Effect of the recombination function on the collection in a p-i-n solar cell, Philos. Mag. 72 (1995) 589-599.

[10] A.V. Shah, F. Sculati-Meillaud, Z.J. Berenyi, O.M. Ghahfarokhi, R. Kumar, Diagnostics of thin-film silicon solar cells and solar panels/modules with variable intensity measurements (VIM), Sol. Energy Mater. Sol. Cells 95 (2011) 398-403.

[11] J. Hubin, A. Shah, E. Sauvain, Effects of dangling bonds on the recombination func tion in amorphous semiconductors, Philos. Mag. Lett. 66 (1992) 115-125.

[12] M. Stuckelberger, A. Shah, J. Krc, M. Despeisse, F. Meillaud, C. Ballif, Internal electric field and fill factor of amorphous silicon solar cells, 35th IEEE Photovoltaic Specialists Conference Proc, 2010, pp. 001569-001574.

[13] A. Shah (Ed.), Thin-film Silicon Solar Cells, CRC Press, 2010.

[14] N. Beck, N. Wyrsch, C. Hof, A. Shah, Mobility lifetime product - a tool for correlating a-Si:H film properties and solar cell performances, J. Appl. Phys. 79 (1996) 9361-9368. 